\title{
A Bayesian Network Approach for Fault-section Estimation
}

\author{
Shu Zhou Xiaoru Wang Qingquan Qian \\ School of Electrical Engineering, Southwest Jiaotong University, Chengdu 610031, P. R. China
}

\begin{abstract}
Changes of the network topology of a power system, which are determined by a sequence of operations of breakers, will influence the relationships between relays and protected sections. Further more, the complexity of power systems and the uncertainty of all kinds of information make the relations between faults and information more complicated and the faultsection estimation problem more difficult. In this paper, the causal relationships among protected sections, relays and breakers are analyzed by using the operation information of relays and breakers. A simple fault-section estimation model based on Bayesian network for power systems is proposed.
\end{abstract}

Keywords: Fault section estimation, Bayesian network, Power systems

\section{Introduction}

The security and reliability of power supply is very important in power system. However, it is inevitable for faults to occur, resulting in unforeseen accidents or abnormal states in the power system. Based on timely fault-section estimation, rapid and correct restoration procedures can thus be taken to minimize the power interruption and limit the impact of outages in the system. So fault-section estimation of power system all along is an important research area, and several kinds of method have been developed, using optimization [1], and artificial neural network [2], information theory [3] and Petri net [4] etc.

The uncertainty existing in fault processing of power system, which is brought about by protective relay failures and/or malfunction, circuit breaker failures and/or malfunction, or communication error etc., makes the fault-section estimation very difficult. As a method of probabilistic inference, Bayesian networks provide a method to represent knowledge in a graphical mode and can be used to do directed graphical description for causal probability relation between random variables. They are usually used for uncertainty knowledge representation, casual inference and diagnosis inference. A component-oriented fault diagnosis model based on Bayesian network composed of Noisy-Or and Noisy-And nodes is proposed in [5], which have rapid inference ability and stronger fault-tolerance ability. In [6], a componentoriented Bayesian network model, in which the temporal order attribute of information is considered, is used for fault diagnosis of power system. In addition, the algorithm to identify the coherence of temporal order information and the method of state estimation for incomplete information are proposed.

A section of power system means a power apparatus, e.g., a transmission or distribution line, bus bar, or transformer, which can be separated from the rest of system by circuit breakers. By the analysis of the causal relationships among sections, protective relays and circuit breakers, we found that the sections are not conditionally independent of each other. So a component-oriented fault diagnosis model is not enough to represent the relations among sections. In this paper, a fault-section estimation approach based on Bayesian network is proposed that is able to deal with the probabilistic causal relationship among section fault, protective relay operation and circuit breaker trip.

\section{Basic of Bayesian network}

A Bayesian network is a directed acyclic graph with probabilistic commentary, in which nodes represent random variables and arcs represent informational or causal dependencies among the variables. The dependencies are quantified by conditional probabilities for each node given its parents in the network.

A Bayesian network $B=\{G, \Theta\}$ is a graphical model that consists of a directed acyclic graph $G$ called the structure and a set of probabilities $\Theta$ defined on $G$. The structure $G=\{X, A\}$ is composed of a finite nonempty set of nodes $X=\left\{X_{1}, X_{2}, \cdots, X_{n}\right\}$ representing random variables and a set of directed arcs $A=\left\{A_{1}, A_{2}, \cdots, A_{e}\right\}$ representing dependencies between variables. Given a set of parent nodes $\mathrm{pa}_{\mathrm{i}}$, the conditional probability of $\mathrm{X}_{\mathrm{i}}=\mathrm{x}_{\mathrm{i}}$ is 
$\theta_{x_{i}, p a_{i}}=p\left(x_{i} \mid p a_{i}\right)$. And the joint probability distribution is represented as: $p(X)=\prod_{i} p\left(X_{i} \mid p a_{i}\right)$

The basic task for probability inference of Bayesian network is to compute the posterior probability distribution for a set of query variables, given exact values for some evidence variables. There are many algorithms for exact probability inference of Bayesian network, such as ploytree propagation algorithm, clique tree propagation algorithm, graph reduction method, combination and optimization method.

\section{Fault-section estimation modeling}

According to the D-Separation criterion of Bayesian network, given the states of protective relays and circuit breakers, sections are not conditionally independent of each other. Constructing a fault-section estimation model based on Bayesian network in a routine way, existing of cycles may destroy the model, or the model will be extraordinary complex.

When faults occur in a power system, the protective relays corresponding to the faulty sections should operate to take the faulty section out of operation. In other words, the faulty sections should be isolated from all operating generators eventually to prevent the faults from being extended. Moreover, it is very rare that many sections suffer from this fault simultaneously or almost simultaneously. Thus, a limited number of small subnetworks will be formed after the operation of protective relays and circuit breakers. Because all faulty sections are included in these small subnetworks, the fault-section estimation problem can be confined to these subnetworks only. In this way, the size of the problem can be greatly reduced.

A simple example as show in Fig. 1 is used to illustrate the principle of fault-section estimation modeling. This example consists of five sections, fifteen protective relays and six circuit breakers. The five sections are bus $\mathrm{A}$, line L1, bus $\mathrm{B}$, line $\mathrm{L} 2$ and bus C. The six circuit breakers are CB1, CB2, CB3, CB4, $\mathrm{CB} 5, \mathrm{CB} 6$. The fifteen protective relays are $\mathrm{Am}, \mathrm{Bm}$, $\mathrm{Cm}, \mathrm{L} 1 \mathrm{Aa}, \mathrm{L} 1 \mathrm{Ba}, \mathrm{L} 2 \mathrm{Ba}, \mathrm{L2Ca}$ L1Ab, L1Bb, L2Bb, L2Cb, L1Ac, L1Bc, L2Bc, L2Cc. Here m, a, b, c identify main protective relays of bus and zone I , zone II, zone III protective relays of line, respectively.

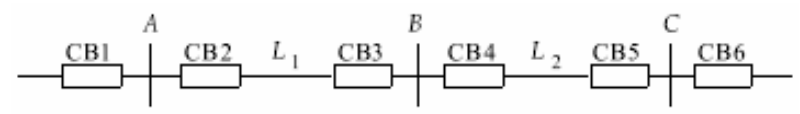

Fig. 1: An simple example of power networks.

\subsection{Probability calculation}

\subsubsection{Prior fault probability of section}

Prior fault probability of a section can be calculated with annual fault frequency $\mu$ of the section [7]. After a section continuously run for a period of time $t$, fault probability of this section equals to the probability of which the fault time interval is $t$.

$$
P(T \leq t)=1-e^{-\omega t}
$$

In Eq. (1), $T$ means the continuous running time of sections without fault. As show in table 1, we can get the prior fault probability of some sections, based on the value of $\omega$ calculated using the data in [8], and $t$ equal to 0.5 on the assumption that there are two maintenances for sections per year.

\begin{tabular}{|c|c|c|c|}
\hline Section & $\begin{array}{c}\text { Line/100 } \\
\mathrm{km}\end{array}$ & Bus/per & $\begin{array}{c}\text { Transformer } \\
/ \mathrm{per}\end{array}$ \\
\hline $\begin{array}{c}\text { Fault } \\
\text { probability }\end{array}$ & 0.1144 & 0.0064 & 0.0048 \\
\hline
\end{tabular}

Table 1: prior fault probability of the protected section

\subsubsection{False operation probability and failure operation probability of protective relay}

False operation probability is equal to the ratio of false operation times to required operation times; For failure operation probability, firstly we need to calculate the average failure operation frequency per year $\mu$, and then calculate failure operation probability using the equation (1). The values of false operation probability and failure operation probability calculated using the data in [8] are shown in table 2.

\begin{tabular}{|c|c|c|c|}
\hline $\begin{array}{c}\text { Fault } \\
\text { probability }\end{array}$ & $\begin{array}{c}\text { Relay of } \\
\text { line/per }\end{array}$ & $\begin{array}{c}\text { Main } \\
\text { relay of } \\
\text { bus/per }\end{array}$ & $\begin{array}{c}\text { Main relay } \\
\text { of } \\
\text { transformer } \\
\text { /per }\end{array}$ \\
\hline $\begin{array}{c}\text { False } \\
\text { operation/\% }\end{array}$ & 0.17 & 0.12 & 0.61 \\
\hline $\begin{array}{c}\text { Failure } \\
\text { operation/\% }\end{array}$ & 0.03 & 1.77 & 0.50 \\
\hline
\end{tabular}

Table 2: false operation probability and failure operation probability

\subsection{Line segmenting and additional node}

Some protective relays can't protect the overall length of a line. So these relays may not operate in principle, when a fault occurred in the line. For example, zone I relay usually protects $80 \%-85 \%$ length of the protected line; Zone II relay can protect the full length of the protected line with no exceeding the 
protective domain of zone I relay of the next line. In this paper, let the protective domain of zone I relay be $80 \%$ length of the protected line. Fig. 2 shows segmenting of line L1 of the simple system shown in Fig. 1 , where segment $1,2,3$ respectively denotes $0 \%$ $20 \%, 20 \%-80 \%, 80 \%-100 \%$ length of line L1. When a fault occurs in segment 1, L1Aa and L1Bb detect the fault and operate to trip appropriate circuit breakers to isolate line $\mathrm{L} 1$ from the rest. It is also the same as L1Aa and L1Ba to segment 2, L1Ab and L1Ba to segment 3 . Using a binary node (0 non-fault, 1 fault) to represent a line in Bayesian network model is not sufficient to embody the protective relationships expatiated. Therefore, a four-number node L1S is added as show in Fig. 3, in which 0 means no fault in L1, 1 means a fault in segment 1, 2 means a fault in segment 2 , and 3 means a fault in segment 3 . The CPT (conditional probability table) of L1S is shown in table 3.

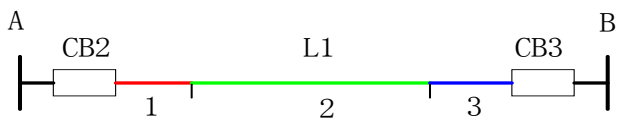

Fig. 2: Segmenting of line L1.

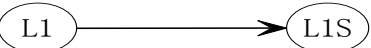

Fig. 3: Additional node L1S.

\begin{tabular}{|c|c|c|}
\hline $\begin{array}{c}\text { CPT of } \\
\text { L1S }\end{array}$ & L1=0 & L1=1 \\
\hline $\mathrm{L} 1 S=0$ & 1 & 0 \\
\hline $\mathrm{L} 1 S=1$ & 0 & 0.2 \\
\hline $\mathrm{L} 1 S=2$ & 0 & 0.6 \\
\hline $\mathrm{L} 1 S=3$ & 0 & 0.2 \\
\hline
\end{tabular}

Table 3: CPT of L1S.

\subsection{Section Model}

According to Bayesian network theory, given the states of protective relays, circuit breakers are Dseparation to sections, that is to say, circuit breakers have no direct causal relationships with sections. Section model is composed of a section node, relay nodes and transitional nodes, in which these relays can detect the fault of the section. Section model of bus B is shown as Fig. 4, as Fig. 5 for line L1.

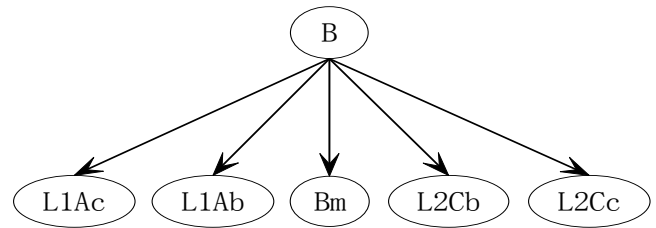

Fig. 4: Section model of B.

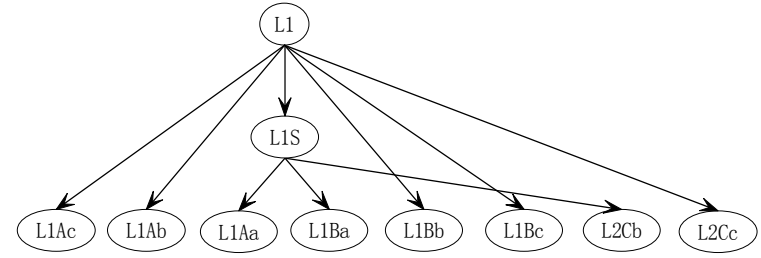

Fig. 5: Section model of L1.

\subsection{Preprocessing}

Operations of breakers change the network topology of a power system that influence the causal relationships between relays and sections. When a breaker tripped, some of relays break away the causal relationships with corresponding sections. The purpose of preprocessing is to identify which relays do not retain causal relationships with this section according to the temporal sequence of breaker operations and to modify the section model accordingly.

Temporal information can be obtained from the SER (Sequence of Events Recording). Based on the network topology of a power system and the operation time-limit settings of relays, the temporal-sequence figures of sections can be constructed. Bus B is taken as an example to explain the temporal-sequence figure shown as Fig. 6. According to the operation time-limit settings of relays, if a fault occurred in $\mathrm{B}$ at the time T0, Bm should operate at T1, L1Ab and L2Cb should operate at T2, L1Ac should operate at T3, and L2Cc should operate at T4, until B was isolated. In Fig. 6, L means the left side of $\mathrm{B}$, to which $\{\mathrm{CB} 1, \mathrm{CB} 2, \mathrm{CB} 3\}$ is corresponding, and $\mathrm{R}$ means the right side of $\mathrm{B}$, to which $\{\mathrm{CB} 4, \mathrm{CB} 5, \mathrm{CB} 6\}$ is corresponding.

Considering the operation temporal sequence of relays corresponding to $\mathrm{B}$, the preprocessing is given under three conditions.

(1) Operation temporal sequence of relays not consistent with the time-limit settings

Lining out breakers in section temporal-sequence figure according to the operation time of these breakers, the relays behind the first breaker are wiped off from the corresponding section model.

For example, if only CB2 of \{CB1, CB2, CB3\} operated and its operation time is between $\mathrm{T} 2$ and $\mathrm{T} 3$, then L1Ac are wiped off from the section model of bus $\mathrm{B}$; for $\{\mathrm{CB} 4, \mathrm{CB} 5, \mathrm{CB} 6\}$, if the operation time of CB4 is before T1 and the operation time of CB5 is between $\mathrm{T} 2$ and $\mathrm{T} 4$, then $\mathrm{L} 2 \mathrm{Cb}$ and $\mathrm{L} 2 \mathrm{Cc}$ are wiped off from the section model of $\mathrm{B}$.

Only if one or more of $\{\mathrm{CB} 1, \mathrm{CB} 2, \mathrm{CB} 3\}$ and one or more of $\{\mathrm{CB} 4, \mathrm{CB} 5, \mathrm{CB} 6\}$ operated respectively, then $\mathrm{Bm}$ is wiped off from the section model of $\mathrm{B}$. At this time, only one node B is kept in the section model, 
and its fault probability is equal to its prior fault probability.

(2) Operation temporal sequence of relays consistent with the time-limit settings

Structure temporal-sequence figures respectively based on the operation time of relays, and deal with them according the processing (1). The principle to determine which model is used is to select the model which consists of minimized relay nodes.

For example, if L1Ab, L1Ac and L2Cc operated, and the temporal sequence of L1Ab and L2Ac is consistent with the time-limit settings shown in Fig. 6, but the temporal sequence of L1Ab and L2Cc is not, then we should structure temporal-sequence figures respectively based on the operation time of L1Ab and L2Cc, and should respectively deal with the temporalsequence figures according the processing expatiated as above (1). The model which consists of minimized relay nodes is kept.

(3) No one relay operated

We don't need to modify the section model in this case.

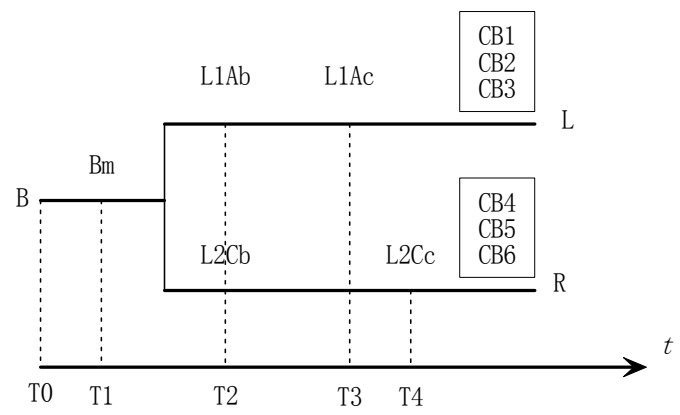

Fig. 6: Temporal-sequence figure of bus B.

\subsection{Fault-section implement}

The real-time network topology determination method is used to identify the network topology of a power system at the normal operation state and postfault operation state respectively. The difference between the two networks topologies gets to the faulty subnetworks. Sections in these small subnetworks are preprocessed and the section models after being preprocessed may consist of the same relay nodes. These section models are combined into a fault-section estimation model. We calculate and reason the faultsection estimation model by using the Bayesian network tool FullBNT developed based on Matlab. Two fault cases of the power system in Fig. 1 are tested, and the results are listed as below.

Test 1: A fault occurred in bus $\mathrm{B}$, relay $\mathrm{Bm}$ operated and tripped the breakers CB3 and CB4. At once another fault occurred in line L1, L1Aa failed in operating, L1Ab operated and tripped CB2. The alarm information is $\mathrm{Bm} \rightarrow \mathrm{CB} 3 、 \mathrm{CB} 4 \rightarrow \mathrm{L} 1 \mathrm{Ab} \rightarrow \mathrm{CB} 2$, where $\rightarrow$ denotes temporal sequence of operations. The faulty subnetwork consists of two sections L1 and $\mathrm{B}$; after the preprocessing of the section models, a fault section estimation model shown as Fig. 7 is obtained. The CPTs of Bm, L1Aa and L1Ab are shown in Table 4. Results of calculation represent that the fault probability of $B$ is 0.8406 and the fault probability of L1 is 0.9383 .
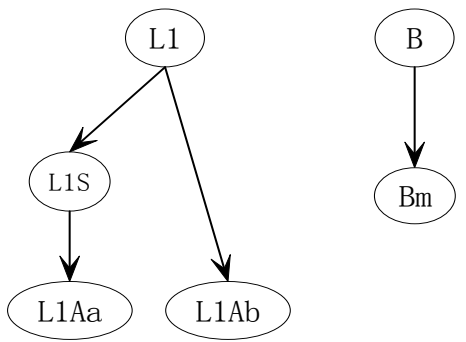

Fig. 7: Fault-section estimation model of test 1.

\begin{tabular}{|c|c|c|}
\hline CPT of $\mathrm{Bm}$ & $\mathrm{B}=0$ & $\mathrm{~B}=1$ \\
\hline $\mathrm{Bm}=0$ & 0.9988 & 0.0177 \\
\hline $\mathrm{Bm}=1$ & 0.0012 & 0.9823 \\
\hline
\end{tabular}

(a)

\begin{tabular}{|c|c|c|c|c|}
\hline $\begin{array}{c}\text { CPT of } \\
\mathrm{L} 1 \mathrm{Aa}\end{array}$ & $\mathrm{L} 1 \mathrm{~S}=0$ & $\mathrm{~L} 1 \mathrm{~S}=1$ & $\mathrm{~L} 1 \mathrm{~S}=2$ & $\mathrm{~L} 1 \mathrm{~S}=3$ \\
\hline $\mathrm{L} 1 \mathrm{Aa}=0$ & 0.9983 & 0.0003 & 0.0003 & 0.9983 \\
\hline $\mathrm{L} 1 \mathrm{Aa}=1$ & 0.0017 & 0.9997 & 0.9997 & 0.0017 \\
\hline
\end{tabular}

(b)

\begin{tabular}{|c|c|c|}
\hline CPT of L1Ab & L1=0 & L1=1 \\
\hline $\mathrm{L} 1 \mathrm{Ab}=0$ & 0.9983 & 0.0003 \\
\hline $\mathrm{L} 1 \mathrm{Ab}=1$ & 0.0017 & 0.9997 \\
\hline
\end{tabular}

(c)

Table 4: CPTs of Bm, L1Aa and L1Ab.

Test 2: A fault occurred in B, Bm operated and tripped CB4, CB3 failed in operating, L1Ab operated and tripped CB2. The alarm information is Bm $\rightarrow$ CB4 $\rightarrow \mathrm{L} 1 \mathrm{Ab} \rightarrow \mathrm{CB} 2$. Fig. 8 shows the fault section estimation model, in which a node N1 is added. The CPTs of $\mathrm{N} 1$ and L1Ab are shown in Table 5. Results of calculation represent that the fault probability of B is 0.9949 and the fault probability of L1 is 0.0298 .

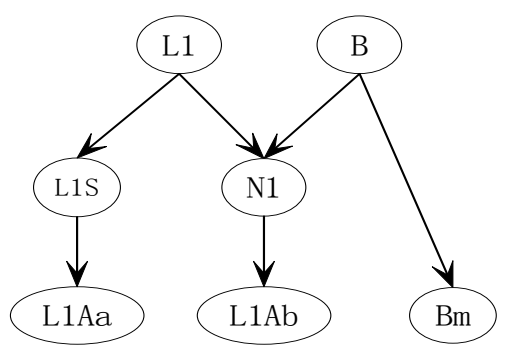

Fig. 8: Fault-section estimation model of test 2. 


\begin{tabular}{|c|c|c|c|c|}
\hline $\begin{array}{c}\text { CPT of } \\
\text { N1 }\end{array}$ & $\begin{array}{c}\mathrm{L} 1=0 \\
\mathrm{~B}=0\end{array}$ & $\begin{array}{c}\mathrm{L} 1=1 \\
\mathrm{~B}=0\end{array}$ & $\begin{array}{c}\mathrm{L} 1=0 \\
\mathrm{~B}=1\end{array}$ & $\begin{array}{c}\mathrm{L} 1=1 \\
\mathrm{~B}=1\end{array}$ \\
\hline $\mathrm{N} 1=0$ & 1 & 0 & 0 & 0 \\
\hline $\mathrm{N}=1$ & 0 & 1 & 1 & 1 \\
\hline
\end{tabular}

(a)

\begin{tabular}{|c|c|c|}
\hline $\mathrm{CPT}$ of $\mathrm{L} 1 \mathrm{Ab}$ & $\mathrm{N} 1=0$ & $\mathrm{~N} 1=1$ \\
\hline $\mathrm{L} 1 \mathrm{Ab}=0$ & 0.9983 & 0.0003 \\
\hline $\mathrm{L} 1 \mathrm{Ab}=1$ & 0.0017 & 0.9997 \\
\hline
\end{tabular}

(b)

Table 5: CPTs of N1 and L1Ab.

\section{Conclusions}

In this paper, line segmenting and additional node are established to make the fault-section model of line more accurate, according to the characteristic of protective reach of line relays. Because the causal relationships among sections, relays and breakers are very complicated, a new method is proposed to deal with these causal relationships by using the operation temporal sequence of relays and breakers, which make the fault-section estimation model simple and have rapid inference ability and stronger fault-tolerance ability. Finally, test results shown that the method proposed is correct and efficient.

\section{References}

[1] F.S. Wen and C.S. Chang, Probabilistic approach for fault-section estimation in power systems based on a refined genetic algorithm, IEE ProcGener. Transm. Distrib., 144:160-168, 1997.

[2] T.S. Bi, Y.X. Ni, F.L. Wu and Q.X. Yang, A novel neural network approach for fault section estimation, Proceedings of the CSEE, 22:73-78, 2002.

[3] L. Tang, H.B. Sun, B.M. Zhang and F. Gao, Online fault diagnosis for power system based on information theory, Proceedings of the CSEE, 23:5-11, 2003.

[4] J.Y. Wang, Y.C. Ji, Q. Chang and L.D. Zhang, Application of petri nets theory to power systems fault diagnosis, Journal Harbin Univ. Sci. \& Tech., 7:77-80, 2002.

[5] L.M. Huo and Y.L. Zhu, Bayesian networks based novel method for fault section estimation of power systems, Journal of North China Electric Power University, 31:30-34, 2004.

[6] X. Wu, C.X. Guo and Y.J. Cao, A new fault diagnosis approach of power system based on Bayesian network and temporal order information, Proceedings of the CSEE, 25:14-18, 2005.
[7] Y.J. Guo, Principle and application of power system reliability, Tsinghua Publishing House, Beijing, 1986.

[8] Y.L. Zhou, Y.L. Wang and M.Y. Zhao, Statistics of operation situation of protective relayings and automation devices of power systems in china in 2004, Power System Technology, 29:42-48, 2005. 